\title{
Fixed point theorems for some generalized nonexpansive mappings in Banach spaces
}

\author{
A. Amini-Harandi
}




\title{
FIXED POINT THEOREMS FOR SOME GENERALIZED NONEXPANSIVE MAPPINGS IN BANACH SPACES
}

\author{
A. AMINI-HARANDI
}

Received 24 September, 2013

\begin{abstract}
In this paper, we first introduce the class of generalized nonexpansive mappings in Banach spaces. This class contains both the classes of nonexpansive and $\alpha$-nonexpansive mappings. In addition, we obtain some fixed point and coincidence point theorems for generalized nonexpansive mappings in uniformly convex Banach spaces. Our results extend some wellknown results in literature.
\end{abstract}

2010 Mathematics Subject Classification: 47H10

Keywords: fixed point, coincidence point, nonexpansive map, uniformly convex space

\section{INTRODUCTION AND PRELIMINARIES.}

Recently, Aoyama and Kohsaka [2] introduced the class of $\alpha$-nonexpansive mappings in Banach spaces and obtained a fixed point theorem for $\alpha$-nonexpansive mappings in uniformly convex Banach spaces. The class of $\alpha$-nonexpansive mappings contains the class of nonexpansive mappings and is related to the classes of firmly nonexpansive mappings and $\lambda$-hybrid mappings in Banach spaces, for more information on firmly nonexpansive mappings and $\lambda$-hybrid mappings see [3], [4], [5], [8], [1] and references therein.

In this paper, we introduce the class of generalized nonexpansive mappings in Banach spaces. This class contains the class of $\alpha$-nonexpansive mappings. In addition, we obtain some fixed point and coincidence point theorems for generalized nonexpansive mappings in uniformly convex and $p$-uniformly convex Banach spaces. Our fixed point theorems generalize some of the results obtained in [2].

In the rest of this section, we recall some definitions and facts which will be used in the next section.

Throughout this paper, every Banach space is real. Let $E$ be a Banach space and let $C$ be a nonempty subset of $E$. We denote the fixed point set of $T$ by $F(T)$. For a Banach space $E$, the norm of $E$ is denoted by $\|$.$\| . Strong convergence of a sequence$

The author was partially supported by a grant from IPM (No. 93470412) and by the Center of Excellence for Mathematics, University of Shahrekord, Iran. 
$\left\{x_{n}\right\}$ in $E$ to $x \in E$ is denoted by $x_{n} \rightarrow x$. For a Banach space $E$, we denote the unit sphere and the closed unit ball centered at the origin of $E$ by $S_{E}$ and $B_{E}$, respectively. We also denote the closed ball with radius $r>0$ centered at the origin of $E$ by $r B_{E}$. Let $E$ be a Banach space with dimension $E \geq 2$. The modulus of convexity of $E$ is the function $\delta_{E}:(0,2] \rightarrow[0,1]$ defined by

$$
\delta_{E}(\epsilon)=\inf \left\{1-\left\|\frac{x+y}{2}\right\|:\|x\|=1,\|y\|=1,\|x-y\| \geq \epsilon\right\} .
$$

A Banach space $E$ is said to be uniformly convex if for each $\epsilon \in(0,2]$, there exists $\delta>0$ such that $\left\|\frac{(x+y)}{2}\right\| \leq 1-\delta$ whenever $x, y \in S_{E}$ and $\|x-y\| \geq \epsilon$. In other words, $E$ is uniformly convex if and only if $\delta_{E}(\epsilon)>0$ for each $\epsilon \in(0,2]$. Every uniformly convex Banach space is reflexive [9].

A Banach space $E$ is called $p$-uniformly convex if there exists a constant $c>0$ such that $\delta_{E}(\epsilon) \geq c \epsilon^{p}$ for all $\epsilon \in(0,2]$. Notice that there is no $p$-uniformly convex Banach space for $p>2$; see, for example [10].

In the sequel we will need the following lemmas.

Lemma 1 ([12]). The Banach space $E$ is uniformly convex if and only if $\|\cdot\|^{2}$ is uniformly convex on bounded convex sets, i.e., for each $r>0$ and $\epsilon \in(0,2 r]$, there exists $\delta>0$ such that

$$
\|t x+(1-t) y\|^{2} \leq t\|x\|^{2}+(1-t)\|y\|^{2}-t(1-t) \delta,
$$

for all $t \in(0,1)$ and for all $x, y \in r B_{E}$ with $\|x-y\| \geq \epsilon$.

Lemma 2 ([11]). Let $1<p \leq 2$ be a given real number. Let $E$ be a $p$-uniformly convex Banach space. Then, there exists a constant $d>0$ such that

$$
\|t x+(1-t) y\|^{p} \leq t\|x\|^{p}+(1-t)\|y\|^{p}-\left(t^{p}(1-t)+t(1-t)^{p}\right) d\|x-y\|^{p},
$$

for all $t \in(0,1)$ and for all $x, y \in E$.

A function $g$ of a nonempty subset $C$ of a Banach space $E$ into $\mathbb{R}$ is said to be coercive if $g\left(z_{n}\right) \rightarrow \infty$ whenever $\left\{z_{n}\right\}$ is a sequence in $C$ such that $\left\|z_{n}\right\| \rightarrow \infty$. Let $l^{\infty}$ denotes the Banach space of bounded real sequences with the supremum norm. It is known that there exists a bounded linear functional $\mu$ on $l^{\infty}$ such that the following three conditions hold:

(1) If $\left\{t_{n}\right\} \in l^{\infty}$ and $t_{n} \geq 0$ for every $n \in \mathbb{N}$, then $\mu\left(\left\{t_{n}\right\}\right) \geq 0$;

(2) If $t_{n}=1$ for every $n \in \mathbb{N}$, then $\mu\left(\left\{t_{n}\right\}\right)=1$;

(3) $\mu\left(\left\{t_{n+1}\right\}\right)=\mu\left(\left\{t_{n}\right\}\right)$ for all $\left\{t_{n}\right\} \in l^{\infty}$.

Such a functional $\mu$ is called a Banach limit and the value of $\mu$ at $\left\{t_{n}\right\} \in l^{\infty}$ is denoted by $\mu_{n} t_{n}$ [9]. Let $\mu$ be a Banach limit and let $\left\{t_{n}\right\} \in l^{\infty}$ be such that $\lim _{n \rightarrow \infty} t_{n}=t$, then the Banach limit of $\left\{t_{n}\right\}$ is also $t$. It is known that the reflexivity of the Banach space $E$ implies the following. 
Lemma 3 ([9]). Let $E$ be a reflexive Banach space, let $C$ be a nonempty, closed, and convex subset of $E$, and let $g: C \rightarrow \mathbb{R}$ be a convex, continuous, and coercive function. Then there exists $u \in C$ such that $g(u)=\inf g(C)$.

Definition 1 ([2]). Let $E$ be a Banach space, let $C$ be a nonempty subset of $E$, and let $\alpha$ be a real number such that $0 \leq \alpha<1$. A mapping $T: C \rightarrow E$ is said to be $\alpha$-nonexpansive if

$$
\|T x-T y\|^{2} \leq \alpha\|T x-y\|^{2}+\alpha\|T y-x\|^{2}+(1-2 \alpha)\|x-y\|^{2}
$$

for all $x, y \in C$.

The following is the main result of Aoyama and Kohsaka [2].

Theorem 1. Let $E$ be a uniformly convex Banach space, let $C$ be a nonempty, closed and convex subset of $E$, and let $T: C \rightarrow C$ be an $\alpha$-nonexpansive mapping for some real number $\alpha$ such that $\alpha<1$. Then $F(T)$ is nonempty if and only if there exists $x \in C$ such that $\left\{T^{n} x\right\}$ is bounded.

\section{FiXed PoINT THEORY}

We first give the definition of generalized nonexpansive mappings.

Definition 2. Let $E$ be a Banach space, and let $C$ be a nonempty subset of $E$. Let $p>1, \alpha_{1} \geq 0, \ldots, \alpha_{m} \geq 0$ with $\sum_{i=1}^{m} \alpha_{i}=1$, and let $a, b, c, d \in \mathbb{R}$ with $b<\alpha_{1}$ for $m=1$ and $b \leq \alpha_{1}$ for $m>1, a+c>0$ and $a+b+c \leq 1$. A mapping $T: C \rightarrow C$ is said to be generalized nonexpansive if

$$
\Sigma_{i=1}^{m} \alpha_{i}\left\|T^{i} x-T^{i} y\right\|^{p} \leq a\|x-y\|^{p}+b\|T y-x\|^{p}+c\|y-T x\|^{p}+d\|x-T x\|^{p}
$$

for all $x, y \in C$.

In the following, we give an example of a generalized nonexpansive mapping which is not an $\alpha$-nonexpansive mapping.

Example 1. Let $E=\mathbb{R}, C=[\sqrt{2}, \sqrt{3}]$ and let $Q$ denotes the set of rational numbers. Let $T: C \rightarrow C$ be defined as

$$
T x= \begin{cases}\sqrt{2}, & x \in Q \\ \sqrt{3} . & x \notin Q\end{cases}
$$

Then $T^{2} x=\sqrt{3}$ for each $x \in C$ and so

$$
\left|T^{2} x-T^{2} y\right|^{2}=0 \leq|x-y|^{2} \text {, for each } x, y \in[\sqrt{2}, \sqrt{3}] .
$$

Thus $T$ is a generalized nonexpansive map. Now, we show that $T$ is not $\alpha$-nonexpansive. On the contrary, assume that there exists $0 \leq \alpha<1$ such that $|T x-T y|^{2} \leq \alpha|T x-y|^{2}+\alpha|T y-x|^{2}+(1-2 \alpha)|x-y|^{2}$, for each $x, y \in[\sqrt{2}, \sqrt{3}]$. 
Let $x \in Q$ and $y \notin Q$ with $\sqrt{2}<x<y<\sqrt{3}$. Then from the above we would have

$$
\begin{gathered}
(\sqrt{3}-\sqrt{2})^{2} \leq \alpha|\sqrt{2}-y|^{2}+\alpha|\sqrt{3}-x|^{2}+(1-2 \alpha)|x-y|^{2} \\
<\alpha(\sqrt{3}-\sqrt{2})^{2}+\alpha(\sqrt{3}-\sqrt{2})^{2}+(1-2 \alpha)(\sqrt{3}-\sqrt{2})^{2}=(\sqrt{3}-\sqrt{2})^{2},
\end{gathered}
$$

a contradiction.

Now, we are ready to state our first main result.

Theorem 2. Let $E$ be a Banach space, let $C$ be a nonempty, closed, and convex subset of $E$, and let $T: C \rightarrow C$ be a generalized nonexpansive mapping. Assume that $E$ is uniformly convex if $p=2$ and assume that $E$ is $p$-uniformly convex for $1<p<2$. Then $\bigcup_{i=1}^{m} F\left(T^{i}\right) \neq \varnothing$ if there exists $x_{0} \in C$ such that $\left\{T^{n} x_{0}\right\}$ is bounded and either $d=0$ or $\lim _{n \rightarrow \infty}\left\|T^{n} x_{0}-T^{n+1} x_{0}\right\|=0$. Moreover, if $\bigcup_{i=1}^{m} F\left(T^{i}\right) \neq \varnothing$ then there exists $x_{0} \in C$ such that $\left\{T^{n} x_{0}\right\}$ is bounded.

Proof. Notice first that if $x_{0} \in \bigcup_{i=1}^{m} F\left(T^{i}\right)$ then there exists $1 \leq j \leq m$ such that $T^{j} x_{0}=x_{0}$ and so $\left\{T^{n} x_{0}: n \in \mathbb{N}\right\}=\left\{T x_{0}, \ldots, T^{j} x_{0}\right\}$. Thus the sequence $\left\{T^{n} x_{0}\right\}$ is bounded. Now assume that there exists $x_{0} \in C$ such that $\left\{T^{n} x_{0}\right\}$ is bounded. Let $\mu$ be a Banach limit and let $y \in C$ be given. For each bounded sequence $\left\{t_{n}\right\} \in l^{\infty}$ the value of $\mu$ at $\left\{t_{n}\right\} \in l^{\infty}$ is denoted by $\mu_{n} t_{n}$. Since $T$ is generalized nonexpansive, we have

$$
\begin{aligned}
& \sum_{i=1}^{m} \alpha_{i}\left\|T^{n+i} x_{0}-T^{i} y\right\|^{p} \\
& \leq a\left\|T^{n} x_{0}-y\right\|^{p}+b\left\|T y-T^{n} x_{0}\right\|^{p} \\
& \quad+c\left\|y-T^{n+1} x_{0}\right\|^{p}+d\left\|T^{n} x_{0}-T^{n+1} x_{0}\right\|^{p},
\end{aligned}
$$

for all $n \in \mathbb{N}$, where $p>1, \alpha_{i} \geq 0, \Sigma_{i=1}^{m} \alpha_{i}=1, b<\alpha_{1}, a+c>0$ and $a+b+c \leq 1$. Since $\mu$ is a Banach limit, we have

$$
\begin{aligned}
& \sum_{i=1}^{m} \alpha_{i} \mu_{n}\left\|T^{n+i} x_{0}-T^{i} y\right\|^{p} \\
& \leq a \mu_{n}\left\|T^{n} x_{0}-y\right\|^{p}+b \mu_{n}\left\|T y-T^{n} x_{0}\right\|^{p} \\
& \\
& \quad+c \mu_{n}\left\|y-T^{n+1} x_{0}\right\|^{p}+d \mu_{n}\left\|T^{n} x_{0}-T^{n+1} x_{0}\right\|^{p} .
\end{aligned}
$$

Thus by our assumptions

$$
\left(\frac{\alpha_{1}-b}{a+c}\right) \mu_{n}\left\|T^{n} x_{0}-T y\right\|^{p}+\Sigma_{i=2}^{m} \frac{\alpha_{i}}{a+c} \mu_{n}\left\|T^{n} x_{0}-T^{i} y\right\|^{p} \leq \mu_{n}\left\|T^{n} x_{0}-y\right\|^{p}
$$

Let $g: C \rightarrow \mathbb{R}$ be a function defined by $g(y)=\mu_{n}\left\|T^{n} x_{0}-y\right\|^{p}$ for all $y \in C$. Now we assert that $g$ is a convex, continuous, and coercive function. The convexity of $g$ follows immediately from Lemmas 1 and 2. We show that $g$ is continuous. Let $\left\{y_{m}\right\}$ be a sequence in $C$ such that $y_{m} \rightarrow y$. Then by the mean value theorem, we have

$$
\left|\left\|T^{n} x_{0}-y_{m}\right\|^{p}-\left\|T^{n} x_{0}-y\right\|^{p}\right|=\left|\left\|T^{n} x_{0}-y_{m}\right\|-\left\|T^{n} x_{0}-y\right\|\right|\left|p c_{m, n}^{p-1}\right|,
$$


for all $m, n \in \mathbb{N}$, where

$$
\min \left\{\left\|T^{n} x_{0}-y_{m}\right\|,\left\|T^{n} x_{0}-y\right\|\right\} \leq c_{m, n} \leq \max \left\{\left\|T^{n} x_{0}-y_{m}\right\|,\left\|T^{n} x_{0}-y\right\|\right\} .
$$

Hence

$$
\begin{aligned}
& \quad\left\|T^{n} x_{0}-y_{m}\right\|^{p}-\left\|T^{n} x_{0}-y\right\|^{p} \mid \\
& \quad \leq\left|\left\|T^{n} x_{0}-y_{m}\right\|-\left\|T^{n} x_{0}-y\right\|\right| p\left(\left\|T^{n} x_{0}-y_{m}\right\|+\left\|T^{n} x_{0}-y\right\|\right)^{p-1} \\
& \quad \leq\left\|y_{m}-y\right\| \sup \left\{p\left(\left\|T^{n} x_{0}-y_{m}\right\|+\left\|T^{n} x_{0}-y\right\|\right)^{p-1}: m, n \in \mathbb{N}\right\},
\end{aligned}
$$

for all $m, n \in \mathbb{N}$. This shows that the function $h: C \rightarrow l^{\infty}$ defined by

$$
h(z)=\left\{\left\|T^{n} x_{0}-z\right\|^{p}\right\}_{n}, \quad z \in C
$$

is continuous. Thus $g=\mu \circ h$ is also continuous. We next show that $g$ is coercive. If $\left\{z_{m}\right\}$ is a sequence in $C$ such that $\left\|z_{m}\right\| \rightarrow \infty$, then we have

$$
\left\|T^{n} x_{0}-z_{m}\right\|^{p} \geq\left(\left|\left\|z_{m}\right\|-\left\|T^{n} x_{0}\right\|\right|\right)^{p}
$$

and hence $g\left(z_{m}\right) \rightarrow \infty$.

It follows from Lemma 3 that there exists $u \in C$ such that $g(u)=\inf g(C)$. Now, we prove that such a point $u$ is unique. Suppose that there exist $u_{1}, u_{2} \in C$ such that $u_{1} \neq u_{2}$ and $g\left(u_{1}\right)=g\left(u_{2}\right)=\inf g(C)$. If $p=2$ then from Lemma 1 for $\epsilon=\left\|u_{1}-u_{2}\right\|>0$, we have $\delta>0$ such that

$$
\left.\left\|\frac{1}{2}\left(T^{n} x_{0}-u_{1}\right)+\frac{1}{2}\left(T^{n} x_{0}-u_{2}\right)\right\|^{2} \leq \frac{1}{2}\left\|T^{n} x_{0}-u_{1}\right\|^{2}+\frac{1}{2} \| T^{n} x_{0}-u_{2}\right) \|^{2}-\delta,
$$

for all $n \in \mathbb{N}$. If $2 \neq p>1$ then from Lemma 2, we get

$$
\begin{aligned}
\| \frac{1}{2}\left(T^{n} x_{0}-u_{1}\right)+ & \frac{1}{2}\left(T^{n} x_{0}-u_{2}\right) \|^{p} \\
& \left.\leq \frac{1}{2}\left\|T^{n} x_{0}-u_{1}\right\|^{p}+\frac{1}{2} \| T^{n} x_{0}-u_{2}\right)\left\|^{p}-\left(\frac{1}{2}\right)^{p} d\right\| u_{1}-u_{2} \|,
\end{aligned}
$$

for all $n \in \mathbb{N}$. The above inequalities imply that $g\left(\frac{u_{1}+u_{2}}{2}\right)<\inf g(C)$. On the other hand, since $\frac{u_{1}+u_{2}}{2} \in C$, we have $\inf g(C) \leq g\left(\frac{u_{1}+u_{2}}{2}\right)$, a contradiction. Hence there exists a unique $u \in C$ such that $g(u)=\inf g(C)$. Now we show that there exists $j \in$ $\{1,2, \ldots, m\}$ such that $g\left(T^{j} u\right) \leq g(u)$. On the contrary, assume that $g(u)<g\left(T^{i} u\right)$, for each $1 \leq i \leq m$. Since by our assumptions $\frac{\alpha_{1}-b}{a+c}+\sum_{i=2}^{m} \frac{\alpha_{i}}{a+c} \geq 1$ then, we get

$$
g(u)<\frac{\alpha_{1}-b}{a+c} g(T u)+\Sigma_{i=2}^{m} \frac{\alpha_{i}}{a+c} g\left(T^{i}\right)
$$

which contradicts (2.1). Hence there exists $j \in\{1,2, \ldots, m\}$ such that $g\left(T^{j} u\right) \leq g(u)$. By the assumption on $T$, we also know that $T^{j} u \in C$, and so $T^{j} u=u$ for some $j \in\{1,2, \ldots, m\}$.

Theorem 2 immediately implies the following corollary. 
Corollary 1. Let $E$ be a uniformly convex Banach space, and let $C$ be a nonempty, closed, and convex subset of $E$. Let $T: C \rightarrow C$ be a mapping satisfying

$$
\|T x-T y\|^{2} \leq a\|x-y\|^{2}+b\|T x-y\|^{2}+c\|x-T y\|^{2}+d\|x-T x\|^{2},
$$

for all $x, y \in C$, where $b<1, a+c>0$ and $a+b+c \leq 1$. Then $F(T)$ is nonempty if there exists $x_{0} \in C$ such that $\left\{T^{n} x_{0}\right\}$ is bounded and either $d=0$ or $\lim _{n \rightarrow \infty} \| T^{n} x_{0}-$ $T^{n+1} x_{0} \|=0$.

The following corollary is a new coincident point result.

Corollary 2. Let $E$ be a uniformly convex Banach space, and let $C$ be a nonempty, closed, bounded and convex subset of $E$. Let $T: C \rightarrow C$ and $S: C \rightarrow C$ be mappings such that $T(C) \subseteq S(C)$ and $S(C)$ is convex and closed. Assume that $T$ and $S$ satisfying

$$
\|T x-T y\|^{2} \leq(1-2 \alpha)\|S x-S y\|^{2}+\alpha\|T x-S y\|^{2}+\alpha\|S x-T y\|^{2},
$$

for all $x, y \in C$, where $0 \leq \alpha<1$. Then $T$ and $S$ have a coincidence point, that is, there exists $u \in C$ such that $T u=S u$.

Proof. We use the technique in [6]. There exists $D \subseteq C$ such that $S(D)=S(C)$ and $S: D \rightarrow C$ is one-to-one. Now, define a map $R: S(D) \rightarrow S(D)$ by $R(S x)=T x$. Since $S$ is one-to-one on $D$ and $T(C) \subseteq S(C), R$ is well-defined. Note that

$$
\begin{aligned}
& \|R(S x)-R(S y)\|^{2}=\|T x-T y\|^{2} \\
& \quad \leq(1-2 \alpha)\|S x-S y\|^{2}+\alpha\|T x-S y\|^{2}+\alpha\|S x-T y\|^{2} \\
& \quad=(1-2 \alpha)\|S x-S y\|^{2}+\alpha\|R(S x)-S y\|^{2}+\alpha\|S x-R(S y)\|^{2}
\end{aligned}
$$

for all $S x, S y \in S(D)$. Since $S(D)=S(C)$ is convex, closed and bounded, by using Corollary $1, R$ has a fixed point in $S(C)$, that is, there exists $u \in C$ such that $R(S u)=S u$, and so $T u=S u$.

Corollary 3. Let $1<p<2, E$ be a p-uniformly convex Banach space, and let $C$ be a nonempty, closed, and convex subset of $E$. Let $T: C \rightarrow C$ be a mapping satisfying

$$
\|T x-T y\|^{p} \leq(1-2 \alpha)\|x-y\|^{p}+\alpha\|T x-y\|^{p}+\alpha\|x-T y\|^{p},
$$

for all $x, y \in C$, where $0 \leq \alpha<1$. Then $F(T)$ is nonempty if and only if there exists $x_{0} \in C$ such that $\left\{T^{n} x_{0}\right\}$ is bounded.

Corollary 4. Let $1<p<2$, E be a p-uniformly convex Banach space, and let $C$ be a nonempty, closed, and convex subset of $E$. Let $T: C \rightarrow C$ be a mapping satisfying

$$
\|T x-T y\|^{p} \leq(1-2 \alpha)\|x-y\|^{p}+\alpha\|T x-y\|^{p}+\alpha\|x-T y\|^{p},
$$

for all $x, y \in C$, where $0 \leq \alpha<1$. Then $F(T)$ is nonempty if and only if there exists $x_{0} \in C$ such that $\left\{T^{n} x_{0}\right\}$ is bounded. 
By the same technique as in the proof of Corollary 2, we can deduce the following coincidence point result from Corollary 4. For some previous studies of coincident point theory, see [7].

Corollary 5. Let $1<p<2, E$ be a p-uniformly convex Banach space, and let $C$ be a nonempty, closed, bounded and convex subset of $E$. Let $T: C \rightarrow C$ and $S: C \rightarrow C$ be mappings such that $T(C) \subseteq S(C)$ and $S(C)$ is convex and closed. Assume that $T$ and $S$ satisfying

$$
\|T x-T y\|^{p} \leq(1-2 \alpha)\|S x-S y\|^{p}+\alpha\|T x-S y\|^{p}+\alpha\|S x-T y\|^{p},
$$

for all $x, y \in C$, where $0 \leq \alpha<1$. Then $T$ and $S$ have a coincidence point, that is, there exists $u \in C$ such that $T u=S u$.

\section{REFERENCES}

[1] K. Aoyama, S. Iemoto, F. Kohsaka, and W. Takahashi, "Fixed point and ergodic theorems for $\lambda$-hybrid mappings in hilbert spaces," J. Nonlinear Convex Anal., vol. 11, pp. 335-343, 2010.

[2] K. Aoyama and F. Kohsaka, "Fixed point theorem for $\alpha$-nonexpansive mappings in banach spaces," Nonlinear Anal., vol. 74, pp. 4387-4391, 2011.

[3] R. E. Bruck and S. Reich, "Nonexpansive projections and resolvents of accretive operators in banach spaces," Houston J. Math., vol. 3, pp. 459-470, 1977.

[4] K. Goebel and W. A. Kirk, Topics in Metric Fixed Point Theory, ser. in: Cambridge Studies in Advanced Mathematics. Cambridge: Cambridge University Press, 1990, vol. 28.

[5] K. Goebel and S. Reich, Uniform Convexity, Hyperbolic Geometry, and Nonexpansive Mappings, ser. in: Monographs and Textbooks in Pure and Applied Mathematics. New York: Marcel Dekker Inc., 1984, vol. 83.

[6] R. H. Haghi, S. Rezapour, and N. Shahzad, "Some fixed point generalizations are not real generalizations," Nonlinear Anal., vol. 74, pp. 1799-1803, 2011.

[7] S. L. Singh and S. N. Mishra, "On a ljubomir Ćirić fixed point theorem for nonexpansive type maps with applications," Indian J. Pure Appl. Math., vol. 33, pp. 531-542, 2002.

[8] R. Smarzewski, "On firmly nonexpansive mappings," Proc. Amer. Math. Soc., vol. 113, pp. 723 $725,1991$.

[9] W. Takahashi, Nonlinear Functional Analysis. Yokohama: Yokohama Publishers, 2000.

[10] Y. Takahashi, H. K., and M. Kato, "On sharp uniform convexity," J. Nonlinear and Convex Analysis, vol. 3, pp. 267-281, 2002.

[11] H. K. Xu, "Inequalities in banach spaces with applications," Nonlinear Anal., vol. 16, pp. 1127$1138,1991$.

[12] Z. Zălinescu, Convex Analysis in General Vector Spaces. $\quad$ River Edge, NJ: World Scientific Publishing Co. Inc., 2002.

Author's address

\section{A. Amini-Harandi}

Department of Mathematic, University of Isfahan, Isfahan, Iran

Current address: School of Mathematics, Institute for Research in Fundamental Sciences (IPM), P.O. Box: 19395-5746, Tehran, Iran

E-mail address: aminih_a@yahoo.com 